\title{
The Relationship of Self-Reported Psychosocial Protective Factors to Characteristics of Adolescent Psychiatric Patients
}

\author{
Shady S Shebak, MD*, Tenzing Yangchen, MD, Katherine Shaver, MS and J Eric Vance, MD \\ Department of Psychiatry, Michigan State University College of Human Medicine, Michigan, USA \\ *Corresponding author: Shady S Shebak, MD, Department of Psychiatry, Michigan State University \\ College of Human Medicine, 15 Michigan St NE, Grand Rapids, MI 49503, USA, Tel: (313)-443-8066
}

\begin{abstract}
Purpose: To explore the relationship of self-reported resiliency (protective) factors on severity of psychiatric characteristics of adolescents hospitalized with psychiatric ilIness.

Methods: Study population included 106 randomly selected youth between ages of 12-17 admitted to the Carilion Child and Adolescent Psychiatric inpatient. Patients completed the Youth Resiliency Checklist (YRC) upon admission. Data was analyzed using one way ANOVA, and correlational tests as appropriate.

Results: A significant relationship was found between the outlooks subscale of the YRC, and length of hospital stay (LOS), $(p=0.0067)$. Also, a significant relationship ( $p=$ 0.0393 ) was found between the social skills subscale, and those with 2 versus 3 or more hospitalizations (means 13.92 versus 11.13). A significant relationship of 0.05 found when comparing social support subscale means for patients with LOS 1-5 days (14.78) to those with LOS 6-10 (12.18).

Conclusion: There are significant relationships between LOS and YRC-rated outlooks and social support, and between number of hospitalizations and YRC-rated social skills.
\end{abstract}

\section{Introduction}

Studies of the phenomenon of psychosocial resilience in the lives of a subset of high-risk individuals have suggested that certain psychosocial protective (resiliency) factors moderate the impact of biopsychosocial risk factors as they contribute to the development of mental illness. Resilience can be defined by factors which are present in high-risk individuals who have escaped illness, but not necessarily present in low-risk individuals who have never been ill. Some studies have provided evidence that certain protective factors can moderate the progression and functional outcome of mental illness in certain populations of adolescents with mental illness. However, it is not clearly established whether, or which psychosocial protective factors might be associated with important features of mental illness in adolescents, including: Outpatient versus inpatient presentation, repeat psychiatric hospitalization, length of stay in hospital, severity of suicidal behaviors, internalizing versus externalizing features of mental ilIness, use of psychotropic medications, and health indicators including obesity and substance use.

This study is an exploratory look at the relationship between self-reported protective factors and features of mental illness and health indicators, in adolescent psychiatric patients. Identification of specific resiliency factors that are associated with features of adolescent psychiatric patients may provide targets for interventions to decrease the severity of illness, service utilization, and improve long-term health outcomes.

We predict that adolescent outpatients with fewer hospitalizations and shorter lengths of stay will have higher overall levels of certain resiliency factors than those with more frequent and longer lengths of stay. We further predict that lower levels of reported protective factors will correlate with more lethal suicidal behavior, greater internalizing disorders, greater use of psychotropic medications, increasing obesity, and greater substance use.

- Aim 1: To de-identify, quantify, and analyze Youth Re-

Citation: Shebak SS, Yangchen T, Shaver K, Vance JE (2020) The Relationship of Self-Reported Psychosocial Protective Factors to Characteristics of Adolescent Psychiatric Patients. Int J Depress Anxiety 2:018. doi.org/10.23937/2643-4059/1710018

Accepted: February 06, 2020: Published: February 08, 2020

Copyright: (c) 2020 Shebak SS, et al. This is an open-access article distributed under the terms of the Creative Commons Attribution License, which permits unrestricted use, distribution, and reproduction in any medium, provided the original author and source are credited. 
siliency Checklist (YRC) information, which has been collected since $6 / 2013$, as a routine part of clinical care and assessment for treatment planning at intake onto the inpatient unit and outpatient clinic for adolescents in the Carilion Child Psychiatry Department.

- Aim 2: To perform retrospective Epic chart review on various characteristics of those patients who have completed a YRC, including: Outpatient or inpatient, frequency of psychiatric hospitalization, length of stay in hospital, severity of suicidal behaviors, internalizing and externalizing features of presenting illness, number of psychotropic medications used, and health indicators including obesity-body mass index (BMI), and substance use.

- Aim 3: To statistically analyze the relationship between resiliency factors as measured on the YRC, and characteristics of these same adolescent psychiatric patients as derived from retrospective Epic chart review.

\section{Significance of Research \& Background}

The significance of this study is to extend existing research on the moderating effects of certain protective and resiliency factors in naturalistic longitudinal studies of resilient high risk individuals, by exploring whether self-reported protective factors are associated with features and severity of psychiatric illness in adolescents.

It is well known that a number of developmental risk factors, also known as childhood adversities, contribute significantly to the development of mental illness, criminality, substance abuse and chronic medical illnesses [1-4]. These psychosocial risk factors have been studied longitudinally in a variety of at-risk populations, and as they accumulate, act to exponentially increase the odds of the development of mental illness and other poor psychosocial and medical outcomes.

In spite of the deleterious effect of risk factors, these same studies of children at risk have revealed that a small subset of youths exposed to significant adversity in childhood do not go on to develop problems (ibid). These youths have been labeled "resilient", and have been noted to possess specific protective (resilience) factors, such as characteristics of personality, family habits, social supports, competencies, life experiences, and outlooks that seem to confer protection in the face of risk.

The study of these protective factors and mechanisms of resilience has great importance for prevention and intervention among high risk populations, since longitudinal studies of child development suggests that protective factors of one type or another, at various stages of life, may intervene to prevent or possibly reverse the onset of mental illness, or other negative psychosocial outcomes, including health problems and serious medical illness. If resilience can be actively built up or enhanced in high risk populations, there is reason to believe it will have salubrious effects on health and well-being.

There is considerable evidence that certain specific protective factors can buffer against certain negative outcomes. For example, in studies of a wide range of negative outcomes, youths who have regular involvement in sports, or other structured extracurricular activities (known protective factors), are less likely to develop cigarette smoking [5], or depression and post-traumatic stress following trauma [6]. Other studies have shown that deliberately building a single protective factor in certain populations of children may mitigate certain bad psychosocial outcomes. Examples include; Big Brother/Big Sister programs providing a mentor (a known protective factor) to children of single parents, and building social problem-solving skills in pre-school children (another known protective factor), both interventions showing longitudinal benefits in preventing the development of problem behaviors $[7,8]$.

This study will use information from the Youth Resiliency Checklist (YRC), a self-report assessment, which has been a routine part of the intake and treatment process in both the outpatient clinic and inpatient unit of Carilion Clinic child psychiatry services since June 2013. Information from the YRC has been used as a routine part of the clinical assessment process, to help physicians, social workers, therapists and other staff working with adolescent patients to understand and integrate the patient's strengths, supports, and protective factors into the treatment planning process. Information from the YRC will be de-identified for purposes of this exploratory stu$\mathrm{dy}$, and protective factors would be analyzed in relation to various characteristics of adolescent psychiatric patients, drawn from retrospective chart review. Other studies have shown that empirically measured resilience factors have shown significant predictive value of certain protective factors in the functional outcomes of seriously mentally ill youths, and in multiply traumatized youths in the juvenile justice system $[6,9,10]$. However, no study to our knowledge has looked at protective factors in relation to adolescent psychiatric outpatients or inpatients.

While this study is an exploratory retrospective study, aimed at identifying significant associations, it is hoped that we might follow-up with prospective assessment of resiliency factors and psychiatric outcomes among adolescents served in our service system to learn how the presence and absence of resiliency factors impacts on course of mental illness, service utilization, and recovery. 


\section{Methods}

Study was approved by the Carilion Clinic Institutional Review Board. Study populations consisted of youths, 12 to 17 year-olds, who were admitted to the Carilion Child and Adolescent Psychiatric Outpatient Clinic, as well as to the $\mathrm{Ca}$ rilion Child and Adolescent Psychiatric Inpatient Unit. Adolescents aged 12-17 were asked to complete a Youth Resiliency Checklist (YRC) upon admission to the inpatient psychiatric services, as a routine clinical assessment, aimed at documenting and focusing on patient strengths and resiliency to assist in treatment planning. The study sample was drawn from admissions to the inpatient services dated from June 2013 to January 2015. Youths younger than 12 at the time of completion of YRC, or those who were unable to read, comprehend, and autonomously fill out the YRC were excluded from the study. We randomly selected 106 charts with completed YRC to review for this study. The numerical ratings of the YRC were entered into a database, which was subsequently cross-referenced to a retrospective chart review on the patients, conducted on EPIC to determine the history of hospitalizations, and other study variables of interest for each study subject, with the reviewer masked to the YRC results. EPIC data reviewed included psychiatric intake or admission assessments (H\&Ps), admission social work assessments on the inpatient unit, medication history, flow sheets for $\mathrm{BMI}, 3$ consecutive psychiatric progress notes, and psychiatric discharge summaries. All YRC and EPIC data was de-identified with a set coding process, and data was aggregated and stratified by variables of interest.

\section{Results}

A significant relationship was found on one way ANOVA between the outlooks subscale of the YRC, and length of hospital stay (LOS), ( $p=0.0067)$. Those with LOS 11 or more days $(N=18)$ had a significantly lower mean outlooks score than those whose LOS was 1 to 5 days ( $N=27$; means 13.39 versus 19.19). Also, a significant relationship ( $p=$ 0.0393 ) was found between the social skills subscale, and those with 2 versus 3 or more hospitalizations (means 13.92 vs. 11.13). Finally, post-hoc Tukey test also revealed significance at the 0.05 level comparing social support subscale means for patients with LOS 1-5 days (14.78) compared to those with LOS 6-10 (12.18). No other significant relationships were found between other YRC scores and suicidal behavior, internalizing or externalizing symptoms, number of psychotropic medications, number of substances used, or body mass index. Table 1 , Table 2 , and Table 3 provide a summary on the significant relationships found in our study.

\section{Discussion}

An inverse relationship was seen between the mean score of outlooks on YRC and the length of stay that was significant with a $p$-value of 0.0067 . This finding supports the thought that patients with a higher sense of self in their ability to cope with stressors and who are more positive in their thought process are less likely to have a longer inpatient hospitalization.

There was a significant difference $(p=0.0393)$ in the mean score of subjectively reported social skills between the groups of patients with 1,2 , or 3 or more numbers of hospitalizations.

Another finding that revealed an inverse relationship was between the mean social support score when compared to length of stay. This was significant with a $p$-value of 0.0533 . Having support networks in the community in the form of teachers, friends, role-models, pets can be protective for the individual that translates to lower duration of hospitalization.

Weak trends were noted between internalizing symptoms when compared to LOS, outlooks and social skills individually. No significant associations or trends were noted on one way ANOVA between any of the subscales of the YRC or the total YRC, in relation to severity of suicidal behavior, externalizing symptoms on admission, number of psychotropic medications at discharge, or number of substances used.

Table 1: Mean outlooks score versus length of stay.

\begin{tabular}{|l|l|}
\hline Mean outlooks score & $\begin{array}{l}\text { Most recent length of } \\
\text { Hospitalization }\end{array}$ \\
\hline 19.2 & $1-5$ days \\
\hline 15.9 & $6-10$ days \\
\hline 13.4 & 11 or more days \\
\hline P-Score & 0.0067 \\
\hline
\end{tabular}

Table 2: Mean social skills score versus number of hospitalizations.

\begin{tabular}{|l|l|}
\hline Mean social Skills Score & Number of Hospitalizations \\
\hline 12.9 & 1 hospitalization \\
\hline 13.9 & 2 hospitalizations \\
\hline 11.1 & 3 or more hospitalizations \\
\hline P-Score & 0.0393 \\
\hline
\end{tabular}

Table 3: Mean social support score versus length of stay.

\begin{tabular}{|l|l|}
\hline Mean social support score & $\begin{array}{l}\text { Most recent length of } \\
\text { Hospitalization }\end{array}$ \\
\hline 14.8 & $1-5$ days \\
\hline 12.2 & $6-10$ days \\
\hline 12.4 & 11 or more days \\
\hline P-Score & 0.0533 \\
\hline
\end{tabular}




\section{Conclusion}

There are significant relationships between LOS and YRC-rated outlooks and social support, and between number of hospitalizations and YRC-rated social skills. This may suggest a moderating effect of these resiliency/protective factors and hospital utilization among adolescent psychiatric patients, and provide potential psychosocial targets for intervention to reduce the burden of hospitalizations.

The YRC checklist can be an insightful tool when used in the inpatient setting. Having an individual fill out this checklist upon admission can be used as a predictive indicator of the potential length of stay, determine need of interventions such as cognitive behavioral therapy to improve any cognitive distortions and poor self-esteem. In the future, we would like to conduct this study on an outpatient population.

\section{References}

1. Anthony EJ (1987) Children at high risk for psychosis growing up successfully. In: Anthony EJ, Cohler BJ, The Invulnerable Child. The Guilford Press, New York and London, 147-184.

2. Luther SS, E Zigler (1991) Vulnerability and competence: A review of research on resilience in childhood. Am J Orthopsychiatry 61: 6-22.

3. Werner EE, Smith RS (1992) Overcoming the odds: High risk children from birth to adulthood. Cornell University Press, New York and London.

4. Felitti VJ, Anda RF, Nordenberg D, Williamson DF, Spitz AM, et al. (1998) Relationship of childhood abuse and household dysfunction to many of the leading causes of death in adults. The adverse childhood experiences (ACES) study. Am J Prev Med 14: 245-258.

5. Anna M Adachi Mejia, Brian A Primack, Michael L Beach, Linda Titus Ernstoff, Meghan R Longacre, et al. (2009) Influence of movie smoking and team sports participation on established smoking. Arch Pediatr Adolesc Med 163: 638643.

6. Rosenberg HJ, Vance JE, Rosenberg SD, Wolford GL, Susan AW, et al. (2012) Trauma exposure, psychiatric disorders and resiliency in juvenile justice involved youth. Psychological trauma: Theory, research, practice, and policy 6: 430-437.

7. Jean BG, Joseph PT, Nancy Resch (1995) Making a difference: An impact study of big brothers/big sisters. Public/ Private Ventures, Philadelphia.

8. Shure MB, Spivack G (1980) Interpersonal problem solving as a mediator of behavioral adjustment in preschool and kindergarten children. JADP 1: 29-44.

9. Vance JE, Bowen NK, Fernandez G, Thompson S (2002) Risk and protective factors as predictors of outcome in adolescents with psychiatric disorder and aggression. J Am Acad Child Adolesc Psychiatry 41: 36-43.

10. Vance JE, Fernandez G, Biber M (1998) Educational progress in a population of youth with aggression and emotional disturbance: The role of risk and protective factors. JEBD 6: 214-221. 